\title{
Dynamic, Multispectral-band IR Thermography Applications in the Petrochemical Furnaces
}

Piotr Pregowski ${ }^{\star a}$, Grzegorz Goleniewski ${ }^{b}$, Wojciech Komosa ${ }^{b}$, Waldemar Korytkowski ${ }^{\text {b }}$, Slawomir Zwolenik

\author{
${ }^{a}$ PIRS Pregowski Infrared Services, Zachodzacego Slonca 36, 01-495 Warsaw, Poland \\ ${ }^{b}$ PKN ORLEN S.A., Dzial Diagnostyki Maszyn, Chemikow 7, 09-411 Plock, Poland \\ ${ }^{c}$ neoVISION, Jasminowa 11, 91-488 Lodz, Poland
}

Keywords: furnace, tubes temperature, flame dynamic, flame visualization, dynamic thermography, multispectral thermography

\section{Abstrakt}

IR radiation thermometry is recognized as the only viable method for measuring temperature distributions in the particularly harsh environments as found in the petrochemical furnaces.

During QIRT'2004 Conference, we presented this idea and the first results of the short, initiating project. At the moment, the next similar project has been executed with its finale planned for the end of March 2008.

This paper will present our innovative method dealing with the flickering and spectrally selective features of the heating mediums, analyzed basing on capturing and elaboration of long sequence of images - instead of the snapshot method. Following to diagnostic aims, a few various narrowband optical filters have been applied. Newly elaborated digital image processing algorithms enable automatic search of a few chosen, eg. statistic values for every pixel of the every frame, with the aim to form substitute images and various types of statistic data saved as txt files. By applying this new methodology, it showed to be possible:

- to prove that heating medium's environment factors influence radiation thermometer's reading more often than not,

- to detect and to remove extremes of fluctuating signals from aerosols and gases - noted even when using a "classic" 3,9/m flame-suppression filter - what lead to obtaining larger and more reliable knowledge about temperature distributions on the tubes or walls inside operating process furnaces;

- to detect, analyze and compare various radiant and dynamic features of the flame and flue gases chich create heating medium inside these furnaces.

Because of meaningful decreasing of the measurement uncertainty in measuring tube's temperature with applying standard $3,91 \mathrm{~m}$ filter, our new method has been routinely used in petrochemical plant in Plock. Numerous examples and comparisons of "classic -raw" single-shot thermograms, and new type of synthesized artificial thermograms will be presented.

The second element of this presentation is focused on looking for a new kind of information about radiant and energetic patterns of the heating medium, i.e. flames and flue gases. These dynamic and volume radiant effects strongly depend on the type of fuel being burnt, type of the furnace's design, etc. and - as very wavelength dependent - not only affect radiation thermometers to different extents... but should offer an additional knowledge about the state of these heating processes. With this aim, sequences of images similar as above - but in the narrow about 100nm bandwidths - matched to the most typical gases as methane, nitrous and carbon oxides, have been collected. In this case, camera calibration and signals interpretations are very specific and much more limited in comparison to tube temperature measurements.

Radiometric model for discussed surveys has to consist of the spectrally and space-dependent components, expressing radiosity of the unknown mixture of gases and aerosols - thus known models and numerical simulations, recognized as still theoretical without proper input data, proved to be of low usability for practice of our investigations. In result, only simple modeling and simulations have been applied for optical filters and recalibration procedure assignments.

Although - due to complexity of the phenomena which decide about our IR camera readings - this kind of investigations is much more qualitative than quantitative yet, we hope to confirm soon some aspects of applied methods as interesting for industrial practice of furnaces diagnostics. 
http://dx.doi.org/10.21611/qirt.2008.06_05_08 